\title{
Predicting the viscosity of alkanes using nonequilibrium molecular dynamics: Evaluation of intermolecular potential models
}

\author{
Richard L. Rowley \\ rowley@byu.edu \\ William Allen
}

Follow this and additional works at: https://scholarsarchive.byu.edu/facpub

Part of the Chemical Engineering Commons

\section{Original Publication Citation}

Allen, William and Richard L. Rowley. "Predicting the viscosity of alkanes using nonequilibrium molecular dynamics: Evaluation of intermolecular potential models." The Journal of Chemical Physics 16 (1997): 1273-1281

\section{BYU ScholarsArchive Citation}

Rowley, Richard L. and Allen, William, "Predicting the viscosity of alkanes using nonequilibrium molecular dynamics: Evaluation of intermolecular potential models" (1997). Faculty Publications. 665.

https://scholarsarchive.byu.edu/facpub/665

This Peer-Reviewed Article is brought to you for free and open access by BYU ScholarsArchive. It has been accepted for inclusion in Faculty Publications by an authorized administrator of BYU ScholarsArchive. For more information, please contact ellen_amatangelo@byu.edu. 


\title{
Predicting the viscosity of alkanes using nonequilibrium molecular dynamics: Evaluation of intermolecular potential models
}

\author{
William Allen and Richard L. Rowley \\ Department of Chemical Engineering, Brigham Young University, Provo, Utah 84602
}

(Received 28 January 1997; accepted 17 March 1997)

\begin{abstract}
Nonequilibrium molecular dynamics (NEMD) viscosity simulations of branched and linear alkanes at liquid densities were performed using both united-atom (UA) and all-atom (AA) intermolecular potential models in order to study the relative efficacy of the models in predicting fluid viscosity. Both models were used in conjunction with fixed bond lengths and bond angles, but different torsional potentials were investigated. The commonly used Ryckaert-Bellemans intermolecular potential model, which accurately predicts viscosities for short straight-chain alkanes, produced values for branched and long-chain alkanes that were significantly below experimental values. Likewise, a more complex UA model that uses transferrable site potentials and is commonly used to simulate thermodynamic properties also under predicted viscosities for branched and long-chain molecules. The UA models were also found to be density dependent, substantially under predicting viscosity at high liquid densities for all model fluids tested. Predicted viscosities using AA intermolecular potential models were generally substantially too large compared to experiment when using model parameters from the literature, even though thermodynamic properties were adequately predicted. However, evidence suggests that accurately modeling the hydrogen interactions and the rotation potential of methyl groups is essential for accurate viscosity simulations. Therefore, a new set of parameters for the hydrogen interactions was regressed using viscosity simulations of 2-methylpropane and $n$-pentane. Like the UA model, the AA model with the new parameters is still somewhat density dependent, but gives reasonably accurate predictions of viscosity for most fluids. (C) 1997 American Institute of Physics. [S0021-9606(97)50224-8]
\end{abstract}

\section{INTRODUCTION}

Liquid viscosity is an important design variable in many industrial applications, yet it is often difficult to predict accurately, especially for a fluid composed of branched molecules. ${ }^{1}$ NEMD simulation provides a method for the calculation of viscosity, theoretically at any condition, from molecular interactions and rigorous physics. The accuracy of the predictions can therefore be viewed as a direct probe of the accuracy of the model used for the interactions.

The majority of NEMD simulations of alkanes to date have used simplified molecular models to study the rheological properties of alkanes at high shear rate..$^{2-8}$ These simulations have in general used Lennard-Jones (LJ), site-site, united-atom (UA) models, where each carbon atom with the hydrogen atoms bonded to it are modeled as a single interacting site. The most common model has been a homogeneous site model due to Ryckaert and Bellemans ${ }^{9}$ (RB), in which all sites are equivalent regardless of the degree of branching. Other simulations have focused on comparing NEMD viscosities extrapolated to zero shear rate to viscosities obtained from equilibrium molecular dynamics (EMD) and the Green-Kubo relation. ${ }^{6,10,11}$ Agreement between the EMD and NEMD methods has provided verification of both methods independent of any inaccuracies in the molecular model.

Only a few NEMD simulations have been performed with the intent of comparing the simulated viscosity to the experimental viscosity. Simulations with the RB model have been used to predict quite accurately the viscosity of $n$-butane, isobutane, $n$-hexane, and cyclohexane over a very wide range of liquid densities. ${ }^{112}$ However, in order to bring the isobutane simulations into agreement with experiment, adjustment of one model parameter was required. This suggests that the homogeneous UA (UA-RB) approach may be inadequate because different model parameters were required for branched and linear groups. ${ }^{13}$ Even with angle bending and bond stretching included in the UA-RB model, simulations on $n$-hexadecane produced viscosities significantly lower than the experimental value, even though the equilibrium hydrostatic pressure was accurately predicted. ${ }^{14}$ This suggests that the UA-RB model is too simple for accurate viscosity predictions for long straight-chain alkanes as well as branched molecules.

Heterogeneous UA models that use different LJ interaction parameters for the methyl and methylene groups have also been used in NEMD simulations of viscosity. Cui et al. used such a heterogeneous model for $n$-decane and found good agreement with experimental data at low liquid densities, ${ }^{10}$ but later simulations at higher densities produced viscosities that were significantly lower than the experimental values. ${ }^{7}$ Simulated viscosities for $n$-hexadecane and $n$-tetracosane were again substantially below the experimental viscosity even though the experimental and simulated $n$-decane values were in reasonably good agreement.

The simulations outlined above seem to indicate that both the homogeneous and heterogeneous UA intermolecular potential models are inadequate for accurately simulating the viscosity of long-chain and branched alkanes, particularly at 
higher densities. In the all-atom (AA) intermolecular potential model, ${ }^{15}$ more realistic asymmetry is provided to the overall intermolecular interactions by placing LJ interaction sites at each atomic center rather than at each carbon. The interaction parameters for this model were regressed from equilibrium properties, ${ }^{14}$ but we know of no systematic study of the use of this model to obtain viscosities from simulations. The objectives of this work were to systematically study the efficacy of different intermolecular potential models in obtaining accurate viscosity information from NEMD simulations, to investigate the cause of any model inadequacies, and to improve on the models where possible.

\section{SIMULATION DETAILS}

\section{A. Model fluids}

Fluids selected for simulation were chosen to represent a variety of branched and linear alkanes. Models representing $n$-butane, $n$-octane, and $n$-dodecane were simulated in order to study model performance for various chain lengths; models for 2-methylpropane, 2,2-dimethylbutane, and 2,2dimethylpropane were studied to determine model effectiveness for branched alkanes. Simulations were also performed using a model representing cyclopentane to see the structural dependence of site-site parameters between straight-chain and ring molecules. Simulations were run at two to three conditions for each fluid in order to determine how the molecular models performed at different densities and temperatures.

\section{B. Potential models}

Pairwise-additive, site-site, LJ interactions were used to model the intermolecular potentials. LJ interaction sites were located at carbon centers for the UA models and at all atomic centers for the AA models. Distances between bonded sites were fixed and constrained to be constant at their equilibrium values as computed by the molecular mechanics program Hyperchem, using the MM+ algorithm. Distances between next-nearest neighbors were also fixed and constrained to be constant to fix all bond angles at their equilibrium values.

Three different intermolecular potential models were used. The homogeneous UA-RB ${ }^{10}$ model was selected because the majority of NEMD viscosity simulations have used this model. A heterogeneous UA mode ${ }^{13}$ (UA-OPLS) was also selected that utilizes transferable intermolecular potentials for alkanes, commonly called optimized potentials of liquid simulations (OPLS). The heterogeneous model used different LJ parameters for carbons with different degrees of branching. The AA model was also an OPLS model with parameters determined by Kaminski et al. ${ }^{15}$ Table I lists the parameters used for the three models. In the table, the number in parentheses next to the UA-OPLS $\mathrm{CH}_{3}$ groups represents the number of carbon atoms bonded to the adjacent carbon atom, which is an indication of the degree of branching on that carbon atom. Cross interaction parameters were calculated using the geometric mean of the individual site parameters. The OPLS parameters shown in Table I were
TABLE I. Intermolecular site-site LJ parameters used in the simulations. Numbers in parentheses indicate the number of carbon atoms bonded to the adjacent carbon atom.

\begin{tabular}{ccll}
\hline \hline Model & Site & $\sigma / \AA$ & $(\epsilon / k) / \mathrm{K}$ \\
\hline UA-RB (Ref. 9) & $\mathrm{All}$ & 3.923 & 72 \\
UA-OPLS (Ref. 11) & $\mathrm{CH}_{3}(4)$ & 3.96 & 72.97 \\
& $\mathrm{CH}_{3}(3)$ & 3.91 & 80.52 \\
& $\mathrm{CH}_{3}(2)$ & 3.905 & 88.07 \\
& $\mathrm{CH}_{2}$ & 3.905 & 59.38 \\
& $\mathrm{CH}$ & 3.85 & 40.26 \\
& $\mathrm{C}$ & 3.80 & 25.16 \\
AA-OPLS (Ref. 13) & $\mathrm{C}$ & 3.5 & 33.21 \\
& $\mathrm{H}$ & 2.5 & 15.10 \\
\hline \hline
\end{tabular}

chosen because the UA and AA parameters were regressed in essentially the same way and at the same conditions. Simulations of the density and heat of vaporization for various fluids have been shown to be essentially equivalent when using the AA-OPLS or UA-OPLS model parameters. ${ }^{15} \mathrm{~A}$ recent comparison showed that UA-OPLS parameters gave excellent predictions of bulk thermodynamic properties for the simple alkanes modeled. ${ }^{16}$ Although the original AAOPLS parameters included small point charges on the hydrogen atoms, simulations using this model indicate that the point charges do not contribute noticeably to the calculation of bulk properties, ${ }^{15,16}$ and they were not included in the AA-OPLS model used in this study.

Intramolecular interactions were modeled using a torsional potential, $u_{\text {tors }}$, assigned to each dihedral angle, $\phi$, in addition to LJ intramolecular interactions between sites separated by four or more carbon bonds. The RB intramolecular potential model

$$
u_{\text {tors }}(\phi) / k=\sum_{i=0}^{5} a_{i} \cos ^{i}(\phi),
$$

was used for dihedral angles involving carbon atoms on a linear portion of a carbon backbone. ${ }^{17}$ For rotations about other dihedral angles (branched carbon backbones and methyl groups), the torsional potential was obtained by fitting the constants of Eq. (1) to potential energies obtained from the molecular mechanics program Hyperchem, using the $\mathrm{MM}+$ algorithm. Table II shows the values used for the constants in Eq. (1) for the various types of dihedral angles. Numbers in parentheses in this table indicate the number of carbon atoms bonded to the specified carbon. We also ran simulations in which branched methyl groups could freely rotate [AA-OPLS (free)], and in which branched methyl groups were fixed rigid in their lowest energy state [AAOPLS (rigid)], to serve as limits on the expected effects of dihedral angles involving hydrogens upon the resultant viscosity predictions. The former limit corresponds to no energy barrier for rotation about the central bond of the sites defining the dihedral angle; the latter corresponds to an infinite energy barrier. 
TABLE II. Constants used in conjunction with Eq. (1) for various torsional potentials. Numbers in parentheses indicate the number of carbon atoms bonded to the specified carbon atom.

\begin{tabular}{lccrrrr}
\hline \hline \multicolumn{1}{c}{ Dihedral angle } & $a_{0}$ & $a_{1}$ & $a_{2}$ & $a_{3}$ & $a_{4}$ & $a_{5}$ \\
\hline H-C (1)-C(2)-C(2) & 792.4 & 2364 & -7.30 & -3139 & 8.34 & -17.32 \\
H-C(1)-C(3)-C(1) & 877.5 & 2613 & -2.21 & -3487 & -1.70 & -0.01 \\
H-C(1)-C(4)-C(1) & 955.3 & 2961 & 8.39 & -3931 & -11.18 & -22.30 \\
H-C(1)-C(4)-C(2) & 1054 & 3140 & -17.62 & -4226 & 12.79 & 44.70 \\
H-C(1)-C(2)-C(4) & 816.6 & 2430 & -71.93 & -3318 & 65.31 & 77.45 \\
H-C(1)-C(4)-C(2) & 959.8 & 2601 & -639.5 & -3852 & 511.6 & 419.0 \\
H-C (1)-C(3)-C(2) & 815.9 & 2648 & 625.3 & -2890 & -500.2 & -698.6 \\
C(1)-C(4)-C(2)-C(1) & 1169 & 3508 & 0.03 & -4678 & -0.02 & 0.02 \\
C(1)-C(4)-C(2)-C(3) & 836.0 & 2511 & 2.64 & -3354 & -2.46 & 6.75 \\
C(1)-C(3)-C(2)-C(4) & 1366 & 2906 & -1010 & -3072 & 621.9 & -111.2 \\
\hline \hline
\end{tabular}

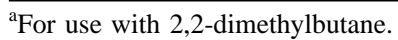

${ }^{\mathrm{b}}$ For use with 2,2,4-trimethylpentane.

\section{Simulation algorithms}

The NEMD NVT simulation algorithm used was that developed by Evans and co-workers. ${ }^{18,19}$ It consists of the SLLOD equations of motion in combination with LeesEdwards "sliding-brick" boundary conditions to generate Couette flow. Temperature was held constant using a Gaussian thermostat. Bond lengths and angles were fixed using Gaussian mechanics to maintain a constant distance between bonded sites and next-nearest neighbors. All UA simulations used 216 molecules; 125 molecules were used for the more CPU intensive AA simulations.

In all simulations, each pair interaction was cut off at a separation distance of $2.8 \sigma_{i j}$. A multiple time step algorithm ${ }^{20}$ was used with a primary cutoff distance of $1.8 \sigma_{i j}$, based upon the center of mass of the molecules. This time-saving technique has been used previously and is adequately described elsewhere. ${ }^{1,12}$ Later simulations used a primary list cutoff of $1.4 \sigma_{i j}$, based upon the closest two sites of interacting molecules. Generally seven steps were performed between full force calculations on the secondary neighbors.

Simulations were performed at four to six different shear rates and extrapolated to zero shear using ${ }^{21-23}$

$$
\eta=\eta_{0}-A \gamma^{1 / 2}
$$

where $\eta$ is viscosity, $\eta_{0}$ is viscosity at zero shear, $\gamma$ is shear rate, and $A$ is an adjustable parameter. The lowest shear rates were about $2 \times 10^{10} \mathrm{~s}^{-1}$ and the highest were about 14 $\times 10^{10} \mathrm{~s}^{-1}$. The range of shear rates corresponded to an optimum found in previous studies for performing the extrapolation. ${ }^{1,12}$ Simulated viscosities at lower shear rates are too noisy and Eq. (2) is known to break down at higher shear rates, either due to pressure effects on the NVT simulation $^{5,6,8}$ or a change in rheology. ${ }^{1}$

There is considerable debate about the best method for extrapolating NEMD viscosities to obtain $\eta_{0}$. While Eq. (2) is the generally accepted method, recent studies ${ }^{5,7}$ suggest that there may be a flat, Newtonian region at very low shear rates when $\ln (\eta)$ is plotted versus $\ln (\gamma)$. However, extremely long simulation times must be used to reduce the uncertainty of the very low-shear viscosities adequately to the point at which such a low-shear plateau can be identified. Moreover, it appears likely that the shear range of any such plateau is a function of the fluid model and the density. We have found that differences arising in $\eta_{0}$ (whether $\eta_{0}$ is obtained from a linear regression on using Eq. (2) or from an average of the values in the flat region on a $\log -\log$ plot) due to the extrapolation method are small relative to the kinds of errors produced by the model itself, and therefore the extrapolation method is not a significant issue for this study on the efficacy of the various intermolecular potential models.

For most of the UA simulations, reduced time steps of 0.0015 were used, corresponding to real time steps of about 2.6-2.9 fs. A reduced time step of 0.001 was used for UA simulations of $n$-dodecane at high temperatures. Reduced time steps of between 0.0005 and 0.001 were used for the AA simulations, corresponding to real times of between 1.15 and 2.3 fs. Property calculations for most UA simulations were performed over a total of about 700-1200 ps at each shear rate. AA simulations were run for less time because of increased computational demands. Approximate running times for AA simulations were about 140 ps for $n$-octane, $170-450$ ps for isobutane, and 800 ps for $n$-butane. Property calculations were divided into five or six equal increments so that block averages and standard deviations could be calculated. Before calculating properties, the simulations were equilibrated at zero shear for 40 to $200 \mathrm{ps}$. The model fluid was then allowed to come to steady state at the shear rate in question for approximately one-sixth of the time over which properties were calculated.

\section{RESULTS AND OBSERVATIONS}

The results obtained from the NEMD simulations using the various models are shown in Table III along with a comparison of simulated and experimental values. ${ }^{24-27}$ Figure 1 summarizes some of these results by model type. In this figure, simulated viscosities for a given fluid and condition are plotted against the values obtained from the UA-OPLS model. Deviations from the $45^{\circ}$ line show differences in the models. For example, the UA-RB and UA-OPLS models are seen to be virtually identical, indicating that distinguishing 
TABLE III. Results of viscosity simulations using various intermolecular potential models.

\begin{tabular}{|c|c|c|c|c|c|c|c|c|c|c|c|c|c|}
\hline \multirow[b]{2}{*}{ Fluid } & \multirow[b]{2}{*}{$T / \mathrm{K}$} & \multirow[b]{2}{*}{$\rho /$ mole $\cdot \mathrm{L}^{-1}$} & \multirow[b]{2}{*}{$\eta_{\exp } / \mathrm{mP}^{\mathrm{a}}$} & \multicolumn{2}{|c|}{ UA-RB } & \multicolumn{2}{|c|}{ UA-OPLS } & \multicolumn{2}{|c|}{ AA-OPLS (rigid) } & \multicolumn{2}{|c|}{ AA-OPLS (Eq. 1) } & \multicolumn{2}{|c|}{ AA-OPLS (free) } \\
\hline & & & & $\eta_{\mathrm{sim}} / \mathrm{mP}$ & $\%$ error & $\eta_{\mathrm{sim}} / \mathrm{mP}$ & $\%$ error & $\eta_{\text {sim }} / \mathrm{mP}$ & $\%$ error & $\eta_{\text {sim }} / \mathrm{mP}$ & $\%$ error & $\eta_{\mathrm{sim}} / \mathrm{mP}$ & $\%$ error \\
\hline$n$-butane & 300 & 9.86 & 1.578 & & & 1.49 & -5.4 & 2.26 & 43.1 & & & 2.00 & 26.8 \\
\hline$n$-butane & 200 & 11.65 & 5.191 & & & 5.21 & 0.3 & 9.19 & 77.0 & & & 7.21 & 38.9 \\
\hline$n$-butane & 150 & 12.42 & 14 & & & 13.3 & -5.0 & 23.6 & 68.2 & & & 17.0 & 21.3 \\
\hline$n$-octane & 423 & 5.102 & 1.683 & 1.43 & -15.0 & 1.50 & -10.7 & & & & & 2.45 & 45.6 \\
\hline$n$-octane & 298 & 6.116 & 5.128 & 4.59 & -10.5 & 4.61 & -10.2 & & & & & 9.96 & 94.2 \\
\hline$n$-octane & 253 & 6.431 & 9.88 & & & 7.33 & -25.8 & & & & & 19.7 & 99.3 \\
\hline$n$-dodecane & 523 & 3.206 & 1.59 & 1.41 & -11.3 & 1.11 & -30.5 & & & & & & \\
\hline$n$-dodecane & 298 & 4.375 & 13.7 & 10.0 & -27.3 & 8.10 & -40.9 & & & & & & \\
\hline cyclopentane & 293 & 10.77 & 4.38 & & & 4.70 & 7.37 & & & & & & \\
\hline cyclopentane & 273 & 10.91 & 5.55 & & & 5.31 & -4.38 & & & & & & \\
\hline cyclopentane & 248 & 11.31 & 7.85 & & & 6.73 & -14.3 & & & & & & \\
\hline 2-methylpropane & 300 & 9.44 & 1.471 & 1.21 & -17.5 & 1.11 & -24.5 & 2.11 & 43.3 & 2.32 & 57.7 & 1.05 & -28.5 \\
\hline 2-methylpropane & 200 & 11.53 & 6.436 & 3.99 & -38.0 & 3.86 & -40.0 & 10.0 & 55.8 & 7.79 & 21.1 & 5.14 & -20.2 \\
\hline 2-methylpropane & 150 & 12.25 & 19.811 & 7.85 & -60.4 & 8.35 & -57.8 & 21.5 & 8.4 & 20.3 & 2.6 & 10.7 & -46.3 \\
\hline 2,2-dimethylpropane & 298 & 8.12 & 2.301 & & & 1.62 & -29.4 & & & & & & \\
\hline 2,2-dimethylpropane & 273 & 8.51 & 3.276 & & & 2.14 & -34.8 & & & & & & \\
\hline 2,2-dimethylpropane & 260 & 8.70 & 4.148 & & & 2.54 & -38.7 & & & & & & \\
\hline 2,2-dimethylbutane & 295 & 7.64 & 3.661 & 2.50 & -31.8 & 3.39 & -7.4 & & & & & & \\
\hline 2,2-dimethylbutane & 270 & 7.95 & 4.931 & 3.52 & -28.6 & 4.43 & -10.1 & & & & & & \\
\hline
\end{tabular}

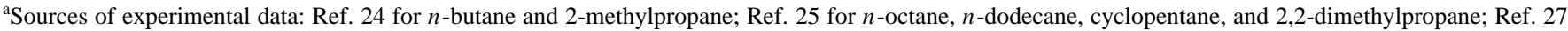
for 2,2-dimethylbutane.

between carbon atoms with different parameters for different degrees of branching will not significantly improve viscosity predictions. It is interesting that viscosities predicted using the AA model are significantly higher than those using the UA models, especially at higher $\eta$ values (generally corresponding to higher densities). Moreover, the difference between the rigid and freely rotating methyl-group predictions

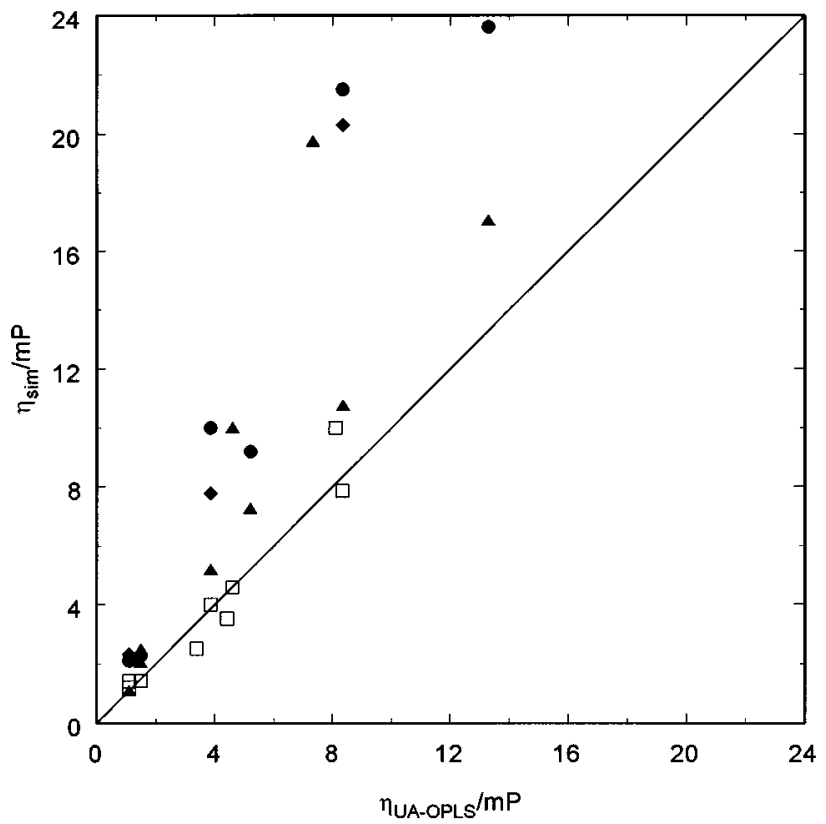

FIG. 1. Sensitivity of viscosity to intermolecular potential model. Deviations of simulated viscosity using UA-RB ( $\square$ ), AA-OPLS (rigid) (O), AAOPLS [Eq. (1)] $(\bullet)$, and AA-OPLS (free) $(\boldsymbol{\Delta})$ models from the UA-OPLS model $\left(45^{\circ}\right.$ line $)$. indicates that the torsional potential for branched molecules can significantly affect simulated viscosities. Again, this difference becomes substantial for the large viscosities and densities. Figure 1 clearly shows that NEMD predictions are much more sensitive to the type of site-site model used than to moderate changes in model parameters.

The usefulness of a molecular simulation approach in predicting (as opposed to correlating) viscosities from sitesite interactions depends upon (1) its ability to use parameters regressed from one property to predict other properties accurately (property independence), (2) its ability to predict a property accurately over a wide range of conditions (density independence), and (3) its ability to use site parameters obtained from one molecule to predict properties for different molecules containing the same sites (structural independence). In what follows, we evaluate the efficacy of the different molecular models from our results in terms of these three criteria.

\section{A. Property independence}

In order to study the relative property independence of the intermolecular site-site potentials and their parameters, NEMD viscosity simulations were performed for the same fluids and at the same conditions used by Jorgensen et al. ${ }^{13}$ to regress the OPLS parameters. This eliminates the influence of density and structure, and focuses the comparison of the results on the property dependence of the models. The results of these simulations are presented in Table IV. Even though the viscosity simulations were performed at the same conditions used to regress the parameters for each fluid, the results show about a $25 \%$ error for the branched fluids using UA-OPLS and about the same magnitude of error for $n$-butane when using the AA-OPLS model (the rigid and 
TABLE IV. Property dependence of the intermolecular potential models.

\begin{tabular}{llrrrrr}
\hline \hline \multicolumn{1}{c}{ Fluid } & \multicolumn{1}{c}{ Model } & $T / \mathrm{K}$ & $\rho / \mathrm{mole} \cdot \mathrm{L}^{-1}$ & $\eta_{\exp } / \mathrm{mP}^{\mathrm{a}}$ & $\eta_{\text {sim }} / \mathrm{mP}$ & $\%$ error \\
\hline$n$-butane & UA-OPLS & 300 & 9.86 & 1.578 & 1.49 & -5.4 \\
$n$-butane & AA-OPLS (rigid) & 300 & 9.86 & 1.578 & 2.26 & 43.1 \\
$n$-butane & AA-OPLS (free) & 300 & 9.86 & 1.578 & 2.00 & 26.8 \\
cyclopentane & UA-OPLS & 293 & 10.77 & 4.380 & 4.70 & 7.4 \\
2-methylpropane & UA-OPLS & 300 & 9.44 & 1.471 & 1.11 & -24.5 \\
2,2-dimethylpropane & UA-OPLS & 298 & 8.12 & 2.301 & 1.62 & -29.4 \\
\hline \hline
\end{tabular}

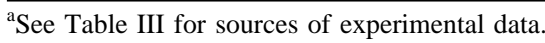

freely rotating models are expected to bracket the value for the actual torsional potential). Reasonable agreement is obtained when using the UA-OPLS model for $n$-butane and cyclopentane, but the implication of these results is that modest errors result from the property dependence in the AA model and in the UA model for branched molecules.

\section{B. Density independence}

To examine the density dependence of the intermolecular potential models, it is desirable to make comparisons at equivalent reduced densities. The packing fraction, $\zeta$, which represents the volume fraction occupied by the molecules themselves, is commonly used for this purpose. ${ }^{28}$ The volume of the molecules was calculated assuming that the volume of each interacting site was given by the volume of a sphere with a diameter of $\sigma_{\text {UA-OPLS }}$, minus the volume of overlap with adjoining sites. Figure 2 shows the results of the density dependence study for the UA and AA models. The densities at which the literature parameters for these models were regressed correspond to a narrow range of $\zeta$, generally $0.47<\zeta<0.54$.

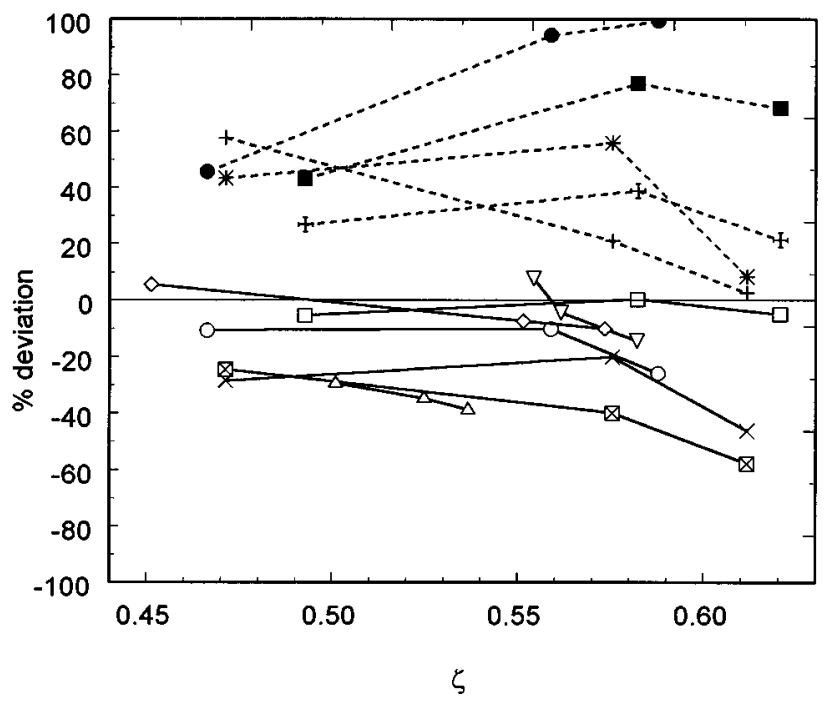

FIG. 2. Percentage deviations of simulated viscosities from experimental values. Shown are UA-OPLS models (solid lines) for $n$-butane $(\square)$, 2-methylpropane $(\otimes)$, 2,2-dimethylpropane $(\triangle)$, cyclopentane, $(\nabla), 2,2-$ dimethylbutane $(\diamond)$, and $n$-octane $(\bigcirc)$; and AA-OPLS models (dashed lines) for $n$-butane (rigid: $\mathbf{\square}$; free: +), 2-methylpropane [rigid: *; Eq. (1): + ; free: $\times]$, and $n$-octane $(\mathbf{\bullet})$.
Figure 2 shows that the UA simulation predictions are in reasonable agreement with experiment for the straight-chain molecules, especially at low density. However, at higher values of $\zeta$, the simulations tend to increasingly underestimate the experimental viscosity for all model molecules. This is especially true for the small branched molecules (isobutane, 2,2-dimethyl-propane, and 2,2-dimethylbutane) and for the longest straight-chain molecule (dodecane). More importantly, the predictions in Fig. 2 exhibit a large downward slope with increasing $\zeta$, indicating that some feature of the actual interactions important to viscosity predictions is not adequately modeled by the UA intermolecular potential model at higher densities.

Figure 2 also shows that the AA model over predicts the experimental viscosity, with the exception of isobutane freely rotating methyl groups. The positive bias of the curves is further evidence of the property dependence discussed above in conjunction with Table IV. This over prediction is especially true for molecules with many fixed hydrogen atoms, such as is the case for the $n$-octane model. Even though the end methyl groups were allowed to rotate freely, the many fixed hydrogens along the carbon backbone of $n$-octane is the most likely cause of the excessively high simulated values.

The distinct grouping of UA and AA predictions in Fig. 2 demonstrates that the addition of hydrogen sites produces a large change in the simulated viscosity, even though both models predict thermodynamic properties quite well. Because of its large property dependence, at least some of the AA model parameters need to be obtained from viscosity data to make the model useful for viscosity predictions. We have therefore regressed a new value for $\sigma_{\mathrm{H}}$ (hydrogen $\mathrm{LJ} \sigma$ ) for use with viscosity simulations. Simulations of $n$-pentane and 2-methylbutane were performed at temperatures of 193.15 and $200 \mathrm{~K}$, respectively, to regress $\sigma_{\mathrm{H}}$. Relatively low temperatures (high densities) were chosen. $n$-Pentane and 2-methylbutane were chosen so as to include both a branched and linear alkane in the regression. The results of six simulations at different shear rates were extrapolated to zero shear rate and compared with experimental values. Based upon this comparison, the values of the $\mathrm{LJ}$ parameters were adjusted for the next iteration. The optimum value obtained for $\sigma_{\mathrm{H}}$ was $\sigma_{\mathrm{H}}=2.38 \AA$. No suitable value of $\sigma_{\mathrm{H}}$ was obtained that would bring the simulated viscosities of both model molecules into agreement with experiment. Increasing $\sigma_{\mathrm{H}}$ increases the simulated viscosity, while de- 


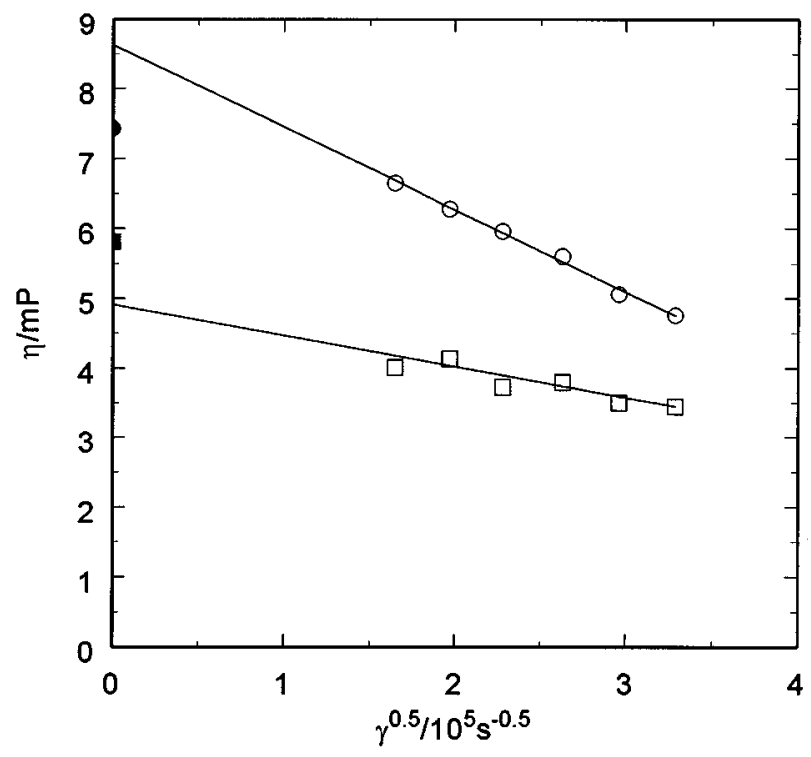

FIG. 3. Optimum value of $\sigma_{\mathrm{H}}$ was obtained using simulated values of $\eta(\gamma)$ for 2-methylpropane $(\square)$ at $200 \mathrm{~K}, \rho=11.356 \mathrm{~mol} / \mathrm{L}$ and $n$-pentane (O) at $193 \mathrm{~K}$, and $\rho=9.928 \mathrm{~mol} / \mathrm{L}$ to extrapolate to zero shear and compare with the experimental values

creasing $\sigma_{\mathrm{H}}$ decreases the viscosity, and the split about the experimental values observed in Fig. 3 could not be significantly decreased. However, with the optimum new value of $\sigma_{\mathrm{H}}$, the prediction for 2-methylpropane is only about $15 \%$ too low, and the predicted viscosity for $n$-pentane only about $15 \%$ too high. Adjustment of $\epsilon_{\mathrm{H}}, \sigma_{\mathrm{C}}$, and $\epsilon_{\mathrm{C}}$ was also attempted, but no combination of parameters was found that would reduce the "split" between predicted and experimental viscosities for these two fluids. Relaxation of the rigid bond length and angle constraints may help reduce this split.

It is evident from Fig. 2 that the UA parameters are less property dependent than the AA parameters. Therefore, to make a better comparison of the density dependence, we redid several of the AA simulations using the new $\sigma_{\mathrm{H}}$ parameter. Results from the AA-OPLS (new) model are shown in Fig. 4, on the same scale as Fig. 2 for comparison purposes. Compared to the AA-OPLS results of Fig. 2, the AAOPLS (new) results are not only in much better agreement with experiment, but they are also less density dependent. Table $\mathrm{V}$ makes a direct comparison between predictions with the AA-OPLS (new) and UA-OPLS models. The average absolute deviation (AAD) of the viscosities shown in Table $\mathrm{V}$ is $13.2 \%$ for the AA-OPLS (new) model compared to $21.3 \%$ for the UA-OPLS model. The improvement is most noticeable for branched molecules and fluids at high density.

The improved results with the AA-OPLS (new) model are an indication that the inclusion of the hydrogen sites in the AA model has a large influence on the predicted viscosity. This is further observed in Figs. 5-7, which show extrapolations of the $\eta(\gamma)$ data to zero shear for simulations of isobutane at three different conditions using five different models. In these figures, it is observed that (1) the UA models increasingly under predict the viscosity at successively

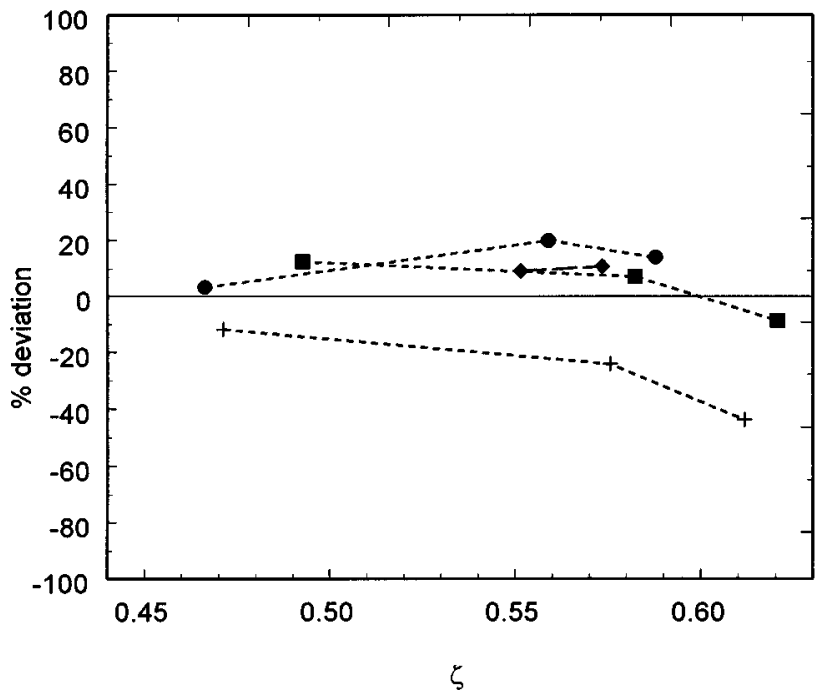

FIG. 4. Percentage deviations of viscosities simulated from experimental values. The AA-OPLS (new) model was used for $n$-butane ( $\mathbf{\square}$ ), 2-methylpropane $(+), n$-octane $(\bullet)$, and 2,2,4-trimethylpentane $(\diamond)$.

higher densities, (2) the AA-OPLS (free) and AA-OPLS (rigid) models in each case bracket the experimental viscosity, with AA-OPLS (free) being more accurate at lower densities and AA-OPLS (rigid) being more accurate at higher densities, (3) the intramolecular potential (free or rigid) effect upon the viscosity increases with density, and (4) the AA-OPLS [Eq. (1)] model results lie between the AA-OPLS (rigid) and AA-OPLS (free) results.

\section{Structural independence}

It is difficult to isolate the structural dependence from the property and density dependence of the models. The effect of chain length in normal alkanes was studied by simulating the viscosities of $n$-butane, $n$-octane, and $n$-dodecane at nearly the same packing fraction, $\zeta$. The UA-OPLS model was used so all three molecules contain the same two sites, $-\mathrm{CH}_{2}$ and $-\mathrm{CH}_{3}$, but with differing numbers of methylene groups. The percent deviations of the simulated viscosity from the experimental value were $-5.4 \%,-10.7 \%$, and $-30.5 \%$, in order of increasing chain length. This indicates that the $-\mathrm{CH}_{2}$ and $-\mathrm{CH}_{3}$ interaction parameters derived from $n$-butane data do not transfer very well to molecules of longer chain length. This conclusion can also be drawn from previous simulations. ${ }^{7,14}$

\section{ANALYSIS OF OBSERVATIONS}

A brief summary of the observations made in this study are:

(1) The property dependence of the UA models is a problem for branched molecules, but not so much for linear and planar alkanes.

(2) The UA-RB and the UA-OPLS models produce quite similar viscosity predictions. 
TABLE V. Comparison of accuracy of simulations using the UA-OPLS and AA-OPLS (new) intermolecular potential models.

\begin{tabular}{|c|c|c|c|c|c|c|c|}
\hline \multirow[b]{2}{*}{ Fluid } & \multirow[b]{2}{*}{$T / \mathrm{K}$} & \multirow[b]{2}{*}{$\rho /$ mole $\cdot \mathrm{L}$} & \multirow[b]{2}{*}{$\eta_{\exp } / \mathrm{mP}^{\mathrm{a}}$} & \multicolumn{2}{|c|}{ UA-OPLS } & \multicolumn{2}{|c|}{ AA-OPLS (new) } \\
\hline & & & & $\eta_{\text {sim }} / \mathrm{mP}$ & $\%$ error & $\eta_{\text {sim }} / \mathrm{mP}$ & $\%$ error \\
\hline$n$-butane & 300 & 9.86 & 1.578 & 1.493 & -5.3 & 1.78 & 12.5 \\
\hline$n$-butane & 200 & 11.65 & 5.191 & 5.21 & 0.3 & 5.56 & 7.1 \\
\hline$n$-butane & 150 & 12.42 & 14.00 & 13.3 & -5.0 & 12.8 & -8.7 \\
\hline$n$-octane & 423 & 5.102 & 1.683 & 1.50 & -10.7 & 1.74 & 3.3 \\
\hline$n$-octane & 298 & 6.116 & 5.128 & 4.61 & -10.2 & 6.15 & 19.9 \\
\hline$n$-octane & 253 & 6.431 & 9.88 & 7.33 & -25.8 & 11.2 & 13.9 \\
\hline$n$-dodecane & 298 & 4.375 & 13.70 & 8.10 & -40.9 & 12.2 & -10.6 \\
\hline 2-methylpropane & 300 & 9.44 & 1.471 & 1.11 & -24.5 & 1.30 & -11.8 \\
\hline 2-methylpropane & 200 & 11.53 & 6.436 & 3.86 & -40.0 & 4.89 & -24.1 \\
\hline 2-methylpropane & 150 & 12.25 & 19.811 & 8.35 & -57.8 & 11.2 & -43.7 \\
\hline 2,2-dimethylbutane & 295 & 7.64 & 3.661 & 3.39 & -7.4 & 3.99 & 9.0 \\
\hline 2,2-dimethylbutane & 270 & 7.95 & 4.931 & 4.43 & -10.1 & 5.46 & 10.8 \\
\hline 2,2,4-trimethylpentane & 348 & 5.64 & 2.818 & 2.08 & -26.2 & 2.78 & -1.3 \\
\hline 2,2,4-trimethylpentane & 298 & 6.02 & 4.718 & 3.28 & -30.5 & 4.56 & -3.4 \\
\hline
\end{tabular}

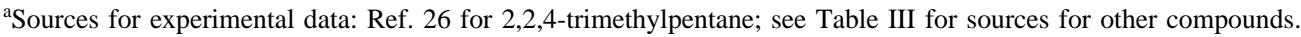

(3) Despite equivalent results for thermodynamic properties, UA-OPLS and AA-OPLS models produce quite different results for simulated viscosities. The AA-OPLS model appears to have a stronger property dependence in that predicted viscosities using literature parameters regressed from equilibrium data were nearly always significantly high.

(4) With the exception of $n$-butane, UA simulations increasingly under predict the viscosity at higher densities, indicating a strong density dependence.

(5) A new $\sigma_{\mathrm{H}}$ parameter regressed from viscosity data greatly improved the accuracy of the AA model and reduced its density dependence.

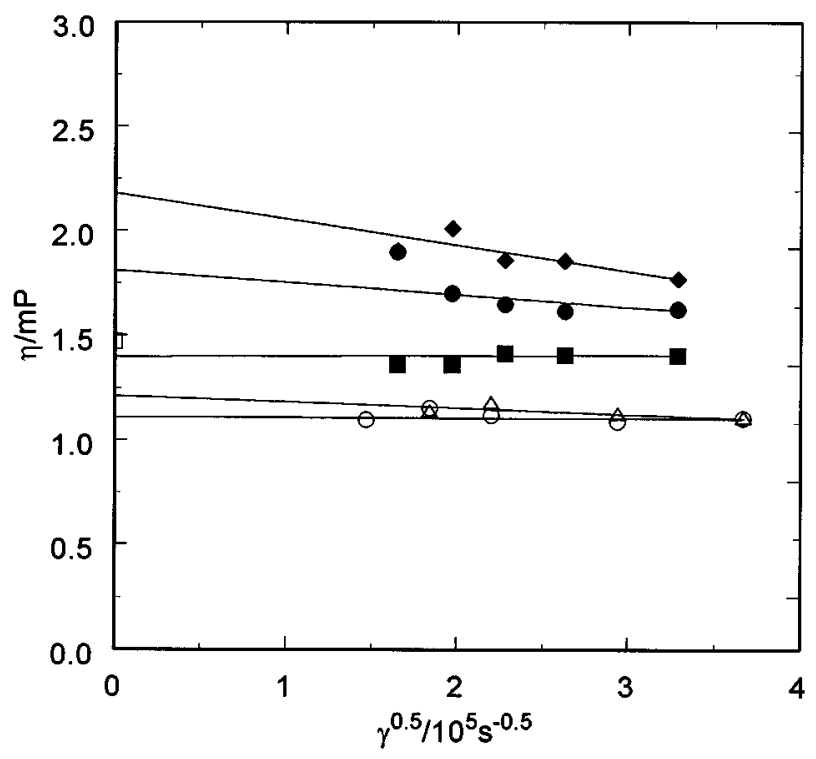

FIG. 5. Extrapolation of simulated 2-methylpropane viscosities to zero shear at $300 \mathrm{~K}$ and $\rho=9.44 \mathrm{~mol} / \mathrm{L}$. Simulated values using the UA-RB $(\triangle)$, UAOPLS (O), AA-OPLS (rigid, $\bullet$ ), AA-OPLS [Eq. (1), ๑], and OPLS (free, 口) models are compared to the experimental value ( $\square$ ).
(6) The torsional potential relating to the rotation of methyl groups on side chains significantly affects the viscosity. At lower densities a freely rotating methyl group models the viscous drag adequately, while fixed methyl groups do better at higher densities. The RB potential was found to represent the torsional potentials adequately along the carbon backbone, and refitting the potential to more accurately model the configurational energies calculated in the computational chemistry package, Hyperchem, produced no change in our results.

Items 3, 5, and 6 imply that accurately modeling hydrogen interactions between molecules affects viscosity simulations more significantly than thermodynamic property calculations. The same effect has been observed previously in the

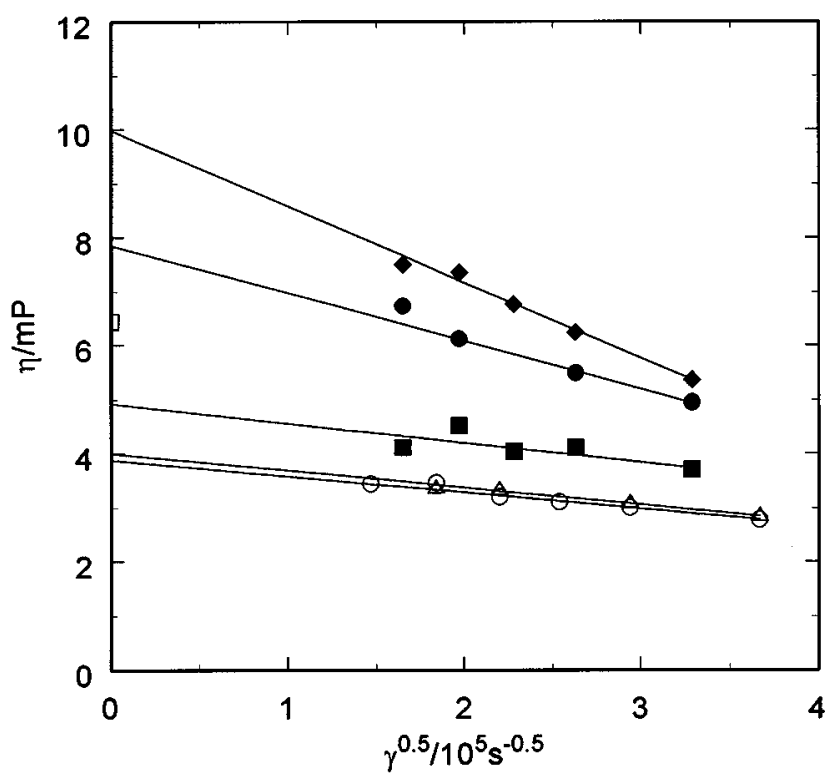

FIG. 6. Extrapolation of simulated 2-methylpropane viscosities to zero shear at $200 \mathrm{~K}$ and $\rho=11.53 \mathrm{~mol} / \mathrm{L}$. Symbols are the same as in Fig. 5 . 


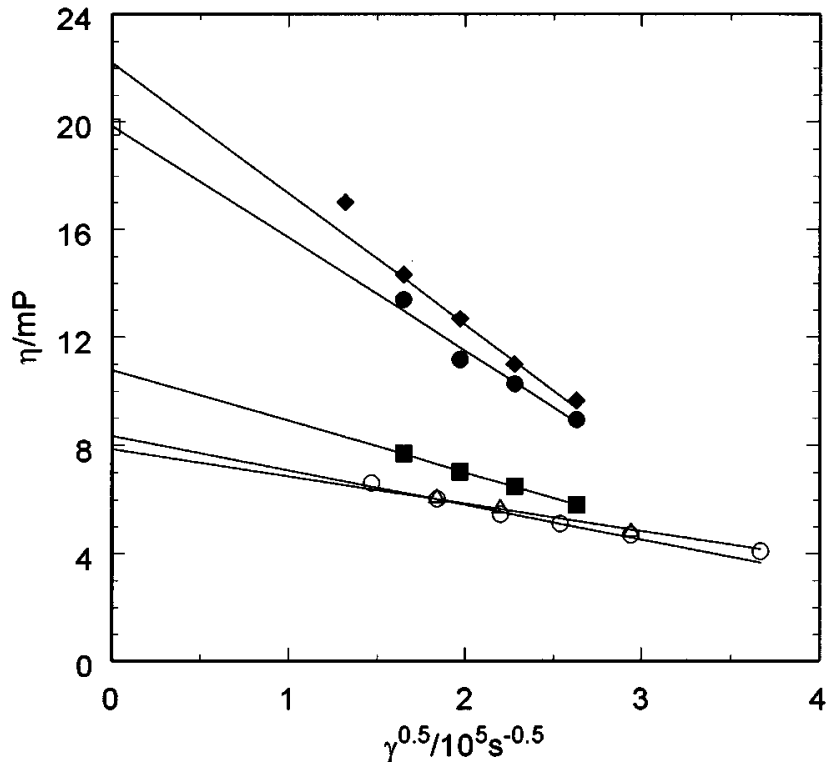

FIG. 7. Extrapolation of simulated 2-methylpropane viscosities to zero shear at $150 \mathrm{~K}$ and $\rho=12.25 \mathrm{~mol} / \mathrm{L}$. Symbols are the same as in Fig. 5 .

study of diffusion coefficients. The Stokes-Einstein equation predicts that viscosity should be roughly inversely proportional to the diffusion coefficient. ${ }^{29}$ Self-diffusion coefficients simulated using AA models of $n$-tridecane were found to be six to eight times smaller than those obtained using UA models. ${ }^{30}$ The authors concluded that "...the different treatment of hydrogens using the [UA] and the [AA] force fields, as represented by the specific force field parameters, has a surprisingly large effect on the dynamic properties as measured by the rate of self diffusion." 30 A similar conclusion was reached by Toxvaerd using an UA model that was made anisotropic to represent the extension of the hydrogen atoms. ${ }^{31}$ This anisotropic model also predicted self-diffusion coefficients significantly lower than the UA model. ${ }^{4}$ It is therefore expected that the inclusion of hydrogen atoms in the model will increase the predicted viscosity over that obtained from UA models.

The observations noted above can be explained in terms of the following hypothesis. Interactions between protruding hydrogen atoms with molecules in different velocity layers cause additional drag on the molecules as they shear past each other, particularly as the density is increased. The UA model, being isotropic about carbon centers, is incapable of modeling this momentum transfer mechanism because it lacks the geometric information concerning the hydrogen atoms. Ability of the methyl group to rotate affects the anisotropy of the protruding hydrogens that cause this additional drag. Energy used to rotate hydrogens out of neighboring shear planes significantly raises the effective viscosity of the fluid. This becomes particularly acute at high densities when this rotation needs to happen to let closely packed molecules shear past each other. Simulations are more accurate for linear and planar molecules than for branched molecules at similar densities, because branched molecules cannot easily align in the shear planes, and therefore the anisotropic poten- tial of the protruding branched atoms would strongly interact at high densities with neighboring molecules in other shear planes. Thermodynamic properties are not as strongly affected by these anisotropic effects, and therefore modeling of the hydrogen atoms is less important. Because the hydrogen atoms have such a strong effect at high densities, lowering $\sigma_{\mathrm{H}}$ slightly in the AA-OPLS (new) model produced much better overall results and corrected the very high predictions that the AA-OPLS model made.

\section{CONCLUSIONS}

We have performed a large number of NEMD NVT simulations on various hydrocarbons using UA-RB, UAOPLS, and AA-OPLS models to study the efficacy of these models in simulating viscosities of real fluids. In particular, we have examined the property, density, and structural dependence of these intermolecular potential models in predicting viscosity. We have also examined the effect that internal torsional potentials have on the predicted viscosity. We found that the UA potentials were nearly equivalent in the ability to predict viscosity. These models are quite structurally dependent and produce poor viscosity results for branched alkanes and molecules with long chains. They are also very density dependent and under predict viscosities of dense liquids. It is hypothesized that these effects are primarily due to a lack of model detail to represent the hydrogen atoms. Hydrogen atom intermolecular potentials have a strong effect on viscosity not observed in the simulation of thermodynamic properties. This accounts for the excessively high viscosity predictions made with the original AA-OPLS model. We regressed a smaller value for $\sigma_{\mathrm{H}}$ to be used in conjunction with the AA-OPLS model. With this value of $\sigma_{\mathrm{H}}$, the AA-OPLS model is capable of reasonably accurate viscosity predictions, and values within about $10-20 \%$ of the experimental can reasonably be expected. Moreover, the AA-OPLS (new) model is less density dependent than either the UA or AA-OPLS models. Nevertheless, the AA models are still density dependent, indicating that there are important viscous drag mechanisms yet missing from the model.

\section{ACKNOWLEDGMENT}

Acknowledgment is made to the donors of The Petroleum Research Fund, administered by the ACS, for partial support of this research.

${ }^{1}$ R. L. Rowley and J. F. Ely, Molec. Phys. 72, 831 (1991).

${ }^{2}$ R. Edberg, G. P. Morris, and D. J. Evans, J. Chem. Phys. 84, 6933 (1986).

${ }^{3}$ R. Edberg, G. P. Morriss, and D. J. Evans, Chem. Phys. 86, 4555 (1987).

${ }^{4}$ P. Padilla and S. Toxvaerd, J. Chem. Phys. 94, 5650 (1991).

${ }^{5}$ P. J. Daivis, D. J. Evans, and G. P. Morriss, J. Chem. Phys. 97, 616 (1992).

${ }^{6}$ P. J. Daivis and D. J. Evans, J. Chem. Phys. 100, 541 (1994).

${ }^{7}$ S. T. Cui, S. A. Gupta, P. T. Cummings, and H. D. Cochran, J. Chem. Phys. 105, 1214 (1996).

${ }^{8}$ G. P. Morriss, P. J. Daivis, and D. J. Evans, J. Chem. Phys. 94, 7420 (1991).

${ }^{9}$ J. P. Ryckaert and A. Bellemans, Discuss. Faraday Soc. 66, 96 (1978).

${ }^{10}$ S. T. Cui, P. T. Cummings, and H. D. Cochran, J. Chem. Phys. 104, 255 (1996).

${ }^{11}$ P. J. Daivis and D. J. Evans, J. Chem. Phys. 103, 4261 (1995). 
${ }^{12}$ R. L. Rowley and J. F. Ely, Molec. Phys. 75, 713 (1991).

${ }^{13}$ W. L. Jorgensen, J. D., Madura, and C. J. Swenson, J. Am. Chem. Soc. 106, 6638 (1984).

${ }^{14}$ S. Chynoweth and Y. Michopoulos, Molec. Phys. 81, 133 (1994).

${ }^{15}$ G. Kaminski, E. M. Duffy, T. Matsui, and W. L. Jorgensen, J. Phys. Chem. 98, 13,077 (1994).

${ }^{16}$ J. Nagy, D. F. Weaver, and V. D. Smith, Jr., J. Phys. Chem. 99, 8058 (1995).

${ }^{17}$ J. P. Ryckaert and A. Bellemans, Chem. Phys. Lett. 30, 123 (1975).

${ }^{18}$ D. J. Evans and G. P. Morriss, Statistical Mechanics of Nonequilibrium Liquids (Academic, New York, 1990).

${ }^{19}$ G. P. Morriss and D. J. Evans, Comp. Phys. Commun. 62, 267 (1991).

${ }^{20}$ W. B. Street, D. J. Tildesley, and G. Saville, Molec. Phys. 35, 639 (1978).

${ }^{21}$ K. Kawasaki and J. D. Gunton, Phys. Rev. A 8, 2048 (1973).

${ }^{22}$ D. J. Evans, H. J. Hanley, and S. Hess, Phys. Today 37, 26 (1984).

${ }^{23}$ P. T. Cummings and D. J. Evans, Ind. Eng. Chem. Res. 31, 1237 (1992).
${ }^{24}$ D. E. Diller and L. J. Van Poolen, Int. J. Thermophys. 6, 43 (1985).

${ }^{25}$ TRC Thermodynamic Tables: Hydrocarbons, Supplement No. 112, edited by M. Frenkel, N. M. Gadalla, K. N. Marsh, and R. C. Wilhoit (Texas A\&M University, College Station, 1996).

${ }^{26}$ J. H. Dymond, N. F. Glen, and J. D. Isdale, Int. J. Thermophys. 6, 233 (1985).

${ }^{27}$ D. S. Viswanath and G. Natarajan, Databook on the Viscosity of Liquids (Hemisphere, New York, 1989).

${ }^{28}$ L. L. Lee, Molecular Thermodynamics of Nonideal Fluids (Butterworths, Boston, 1988).

${ }^{29}$ R. C. Reid, J. M. Prausnitz, and B. E. Poling, Properties of Gases and Liquids, 4th edition (McGraw-Hill, New York, 1987).

${ }^{30}$ D. Y. Yoon, G. D. Smith, and T. Matsuda, J. Chem. Phys. 98, 10,037 (1993).

${ }^{31}$ S. Toxvaerd, J. Chem. Phys. 93, 4290 (1990). 